\title{
Upright Cone CT of the hindfoot: Comparison of the non-weight-bearing with the upright weight-bearing position
}

\author{
Hirschmann, Anna ; Pfirrmann, Christian W A ; Klammer, Georg ; Espinosa, Norman ; Buck, Florian \\ $\mathrm{M}$
}

\begin{abstract}
OBJECTIVES: To prospectively compare computed tomography (CT) of the hindfoot in the supine non-weight-bearing position (NWBCT) with upright weight-bearing position (WBCT). METHODS: Institutional review board approval and informed consent of all patients were obtained. NWBCT and WBCT scans of the ankle were obtained in 22 patients (mean age, $46.0 \pm 17.1$ years; range 19-75 years) using a conventional 64-row CT for NWBCT and a novel cone-beam CT for WBCT. Two musculoskeletal radiologists independently performed the following measurements: the hindfoot alignment angle, fibulocalcaneal and tibiocalcaneal distances, lateral talocalcaneal joint space width, talocalcaneal overlap and naviculocalcaneal distance. Significant changes between NWBCT and WBCT were sought using Wilcoxon signed-rank test. $\mathrm{P}$ values $<0.05$ were considered statistically significant. RESULTS: Significant differences were found for all measurements except the hindfoot alignment angle and tibiocalcaneal distance. Significant measurement results were as follows (NWBCT/WBCT reader 1; NWBC$\mathrm{T} / \mathrm{WBCT}$ reader 2 , mean \pm standard deviation): fibulocalcaneal distance $3.6 \mathrm{~mm} \pm 5.2 / 0.3 \mathrm{~mm} \pm 6.0$ $(\mathrm{P}=0.006) ; 1.4 \mathrm{~mm} \pm 6.3 /-1.1 \mathrm{~mm} \pm 6.3(\mathrm{P}=0.002)$, lateral talocalcaneal joint space width $2.9 \mathrm{~mm} \pm$ $1.7 / 2.2 \mathrm{~mm} \pm 1.1(\mathrm{P}=0.005) ; 3.4 \mathrm{~mm} \pm 1.9 / 2.4 \mathrm{~mm} \pm 1.3(\mathrm{P}=0.001)$, talocalcaneal overlap $4.1 \mathrm{~mm} \pm$ $3.9 / 1.4 \mathrm{~mm} \pm 3.9(\mathrm{P}=0.001) ; 4.5 \mathrm{~mm} \pm 4.3 / 1.4 \mathrm{~mm} \pm 3.7(\mathrm{P}<0.001)$ and naviculocalcaneal distance $13.5 \mathrm{~mm} \pm 4.0 / 15.3 \mathrm{~mm} \pm 4.7(\mathrm{P}=0.037) ; 14.0 \mathrm{~mm} \pm 4.4 / 15.7 \mathrm{~mm} \pm 6.2(\mathrm{P}=0.100)$. Interreader agreement was good to excellent (ICC 0.48-0.94). CONCLUSION: Alignment of the hindfoot significantly changes in the upright weight-bearing CT position. Differences can be visualised and measured using WBCT. KEY POINTS: • Cone-beam computed tomography (CBCT) offers new opportunities for musculoskeletal problems - Visualization and quantification of hindfoot alignment are possible in upright weight-bearing CBCT • Hindfoot alignment changes significantly from non-weight-bearing to weight-bearing CT • The weight-bearing position leads to a decrease in the fibulocalcaneal distance and talocalcaneal overlap • The naviculocalcaneal distance is increased in the weight-bearing position.
\end{abstract}

DOI: https://doi.org/10.1007/s00330-013-3028-2

Posted at the Zurich Open Repository and Archive, University of Zurich

ZORA URL: https://doi.org/10.5167/uzh-89427

Journal Article

Published Version

Originally published at:

Hirschmann, Anna; Pfirrmann, Christian W A; Klammer, Georg; Espinosa, Norman; Buck, Florian M (2014). Upright Cone CT of the hindfoot: Comparison of the non-weight-bearing with the upright weight-bearing position. European Radiology, 24(3):553-558. 
DOI: https://doi.org/10.1007/s00330-013-3028-2 


\title{
Upright Cone CT of the hindfoot: Comparison of the non-weight-bearing with the upright weight-bearing position
}

\author{
Anna Hirschmann - Christian W. A. Pfirrmann • \\ Georg Klammer • Norman Espinosa • Florian M. Buck
}

Received: 24 July 2013 / Accepted: 9 September 2013 / Published online: 26 September 2013

(C) European Society of Radiology 2013

\begin{abstract}
Objectives To prospectively compare computed tomography (CT) of the hindfoot in the supine non-weight-bearing position (NWBCT) with upright weight-bearing position (WBCT).

Methods Institutional review board approval and informed consent of all patients were obtained. NWBCT and WBCT scans of the ankle were obtained in 22 patients (mean age, 46.0土 17.1 years; range 19-75 years) using a conventional 64-row CT for NWBCT and a novel cone-beam CT for WBCT. Two musculoskeletal radiologists independently performed the following measurements: the hindfoot alignment angle, fibulocalcaneal and tibiocalcaneal distances, lateral talocalcaneal joint space width, talocalcaneal overlap and naviculocalcaneal distance. Significant changes between NWBCT and WBCT were sought using Wilcoxon signed-rank test. $P$ values $<0.05$ were considered statistically significant.

Results Significant differences were found for all measurements except the hindfoot alignment angle and tibiocalcaneal distance. Significant measurement results were as follows (NWBCT/ WBCT reader 1; NWBCT/WBCT reader 2, mean \pm standard deviation): fibulocalcaneal distance $3.6 \mathrm{~mm} \pm 5.2 / 0.3 \mathrm{~mm} \pm 6.0$ $(P=0.006) ; 1.4 \mathrm{~mm} \pm 6.3 /-1.1 \mathrm{~mm} \pm 6.3 \quad(P=0.002)$, lateral talocalcaneal joint space width $2.9 \mathrm{~mm} \pm 1.7 / 2.2 \mathrm{~mm} \pm 1.1$ $(P=0.005) ; 3.4 \mathrm{~mm} \pm 1.9 / 2.4 \mathrm{~mm} \pm 1.3(P=0.001)$, talocalcaneal overlap $4.1 \mathrm{~mm} \pm 3.9 / 1.4 \mathrm{~mm} \pm 3.9(P=0.001) ; 4.5 \mathrm{~mm} \pm 4.3 /$ $1.4 \mathrm{~mm} \pm 3.7(P<0.001)$ and naviculocalcaneal distance
\end{abstract}

\footnotetext{
A. Hirschmann $(\bowtie) \cdot$ C. W. A. Pfirmann • F. M. Buck Department of Radiology, Orthopaedic University Hospital Balgrist, University of Zurich, Forchstrasse 340, 8008 Zurich, Switzerland e-mail: anna.hirschmann@balgrist.ch

G. Klammer $\cdot$ N. Espinosa

Department of Orthopedic Surgery, Orthopaedic University Hospital Balgrist, University of Zurich, Forchstrasse 340, 8008 Zurich, Switzerland
}

$13.5 \mathrm{~mm} \pm 4.0 / 15.3 \mathrm{~mm} \pm 4.7(P=0.037) ; 14.0 \mathrm{~mm} \pm 4.4 /$ $15.7 \mathrm{~mm} \pm 6.2(P=0.100)$. Interreader agreement was good to excellent (ICC 0.48-0.94).

Conclusion Alignment of the hindfoot significantly changes in the upright weight-bearing CT position. Differences can be visualised and measured using WBCT.

Key Points

- Cone-beam computed tomography (CBCT) offers new opportunities for musculoskeletal problems

- Visualization and quantification of hindfoot alignment are possible in upright weight-bearing $C B C T$

- Hindfoot alignment changes significantly from non-weightbearing to weight-bearing $C T$

- The weight-bearing position leads to a decrease in the fibulocalcaneal distance and talocalcaneal overlap

- The naviculocalcaneal distance is increased in the weight-bearing position

Keywords Hindfoot alignment · Cone-beam computed tomography · Weight-bearing · Talocalcaneal overlap . Fibulocalcaneal distance

\section{Introduction}

Hindfoot alignment has been well investigated on conventional weight-bearing radiographs. However, considering the complex anatomy and relationship of the foot joints, any two-dimensional depiction of bone deformities and its capability of true assessment will reach certain limitations [1-5]. Until now, computed tomography (CT) of the lower extremity has only been feasible using conventional $\mathrm{CT}$ in the supine position. Exertion of axial load to the joints using special apparatuses may simulate weight-bearing conditions but have only been applied in the supine position [6-9]. As such, the results of those investigations can be questioned because they 
do not replicate natural forces running across the foot and ankle [3, 4, 6-11]. More recently, a novel fluoroscopy system has been shown to provide quite accurate $3 \mathrm{D}$ reconstructions of the foot in an upright position [12]. However, the novel cone-beam CT (Planmed Verity Extremity, Planmed Oy, Helsinki, Finland) allows $\mathrm{CT}$ of the ankle and foot to be performed in the upright weight-bearing position [13, 14]. $\mathrm{CT}$ allows precise visualisation of the hindfoot and therefore seems superior to conventional radiographs in the assessment of hindfoot alignment $[12,15]$. To our knowledge, there is no comparison of hindfoot alignment using non-weight-bearing and upright weight-bearing CT. The purpose of this study was to prospectively quantify changes in alignment of the hindfoot between non-weight-bearing (NWBCT) and upright weightbearing $\mathrm{CT}$ (WBCT).

\section{Materials and methods}

\section{Patient and CT technique}

Institutional review board approval and informed consent of all patients were obtained. NWBCT and WBCT of the ankle were performed in 22 patients (mean age, $46.0 \pm 17.1$ years; range, 19-75 years; 8 women; mean age, 51.0 years; range, 19-69 years; 14 men; mean age, 43.2 years; range, 2575 years) using a conventional 64-row CT unit (Brilliance 64, Philips Healthcare, Best, The Netherlands) for NWBCT and a novel cone-beam extremity CT for WBCT (Planmed Verity Extremity, Planmed Oy, Helsinki, Finland). Imaging was performed on the same day. In the supine position the ankle was examined in a neutral position; a strap around the foot and a foam pad on the sole were used to prevent motion artefacts. For the upright CT examination, the foot was placed in a neutral position on a dedicated platform; the other foot rested on the gantry (Fig. 1). Axial images (NWBCT: tube voltage, $120 \mathrm{kV}$; tube current, $100 \mathrm{mAs} / \mathrm{slice}$; pitch factor, $0.352 ; \mathrm{CTDI}_{\mathrm{vol}} 7.6 \mathrm{mGy}$; matrix, $512 \times 512$; reconstruction thickness, $0.67 \mathrm{~mm}$; reconstruction increment, $0.33 \mathrm{~mm}$; WBCT: tube voltage, $96 \mathrm{kV}$; tube current, $7.5 \mathrm{mAs}$; $\mathrm{CTDI}_{\mathrm{vol}}$ $4.3 \mathrm{mGy}$; matrix, $160 \times 160 \times 130$; pixel size, $0.4 \mathrm{~mm}$; slice interval $0.4 \mathrm{~mm}$ ) of the ankle joint were acquired. Axial (reconstruction thickness $2 \mathrm{~mm}$ ), sagittal (reconstruction thickness $2 \mathrm{~mm}$ ) and coronal (reconstruction thickness $1 \mathrm{~mm}$ ) bone window reformations of these images were used for measurements.

All patients included in this study were referred from the orthopaedic department for a CT scan of the ankle. Indications for the CT were osteoarthritis of the hindfoot $(n=8)$, osteochondral defects of the talus $(n=6)$, evaluation of foot pain $(n=5)$ and others $(n=3)$, including healed fracture of the cuboid $(n=1)$ and osteonecrosis of the navicular $(n=2)$. Patients were included in this study if they were able to fully bear

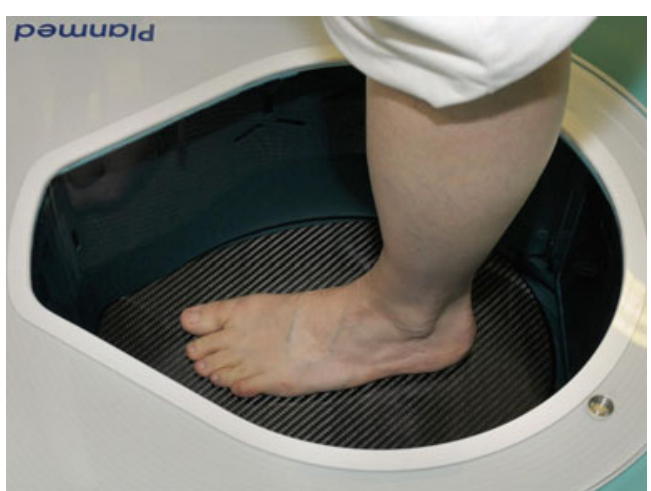

Fig. 1 Photograph of the position during upright weight-bearing foot examination using the cone-beam CT scanner

weight on the examined foot. Patients were excluded if they had undergone a prior foot surgery, if they were under the age of 18 and if they did not give informed consent.

\section{Analysis of CT}

Two musculoskeletal radiologists independently performed the following measurements on NWBCT and WBCT: the hindfoot alignment angle; fibulocalcaneal and tibiocalcaneal distances and lateral talocalcaneal joint space width in the coronal plane; talocalcaneal overlap in the coronal plane and naviculocalcaneal distance in the sagittal plane.

The hindfoot alignment angle was based on the axis of the distal tibial defined by a perpendicular line to the distal tibial joint surface and a line paralleling the medial osseous contour of the calcaneus on the most posterior image including the tibia and calcaneus (Figs. 2 and 3) [2, 16, 17]. A positive hindfoot alignment angle indicated valgus, and a negative angle indicated varus.

For the fibulocalcaneal and tibiocalcaneal distances as well the lateral talocalcaneal joint space width, the coronal image with the lowest extension of the fibular tip was used. The reference line was drawn parallel to the calcaneal surface (Fig. 4). Distances from this line were measured to the fibular tip (fibulocalcaneal distance) and to the tip of the medial malleolus (tibiocalcaneal distance) (Fig. 4). Distances below the line were defined as negative, above the line as positive. The lateral talocalcaneal joint space width was measured (Fig. 4).

For the talocalcaneal overlap, a coronal slice through the midpoint of the anterior facet of the subtalar joint was chosen measuring the length of the talus overlapping the calcaneus along the calcaneal joint surface (Fig. 5). A positive value indicated talocalcaneal overlap; a negative value indicated no overlap. The naviculocalcaneal distance was assessed measuring the shortest distance from the navicular bone to the calcaneus at the level below the talar head on sagittal images (Fig. 6). 


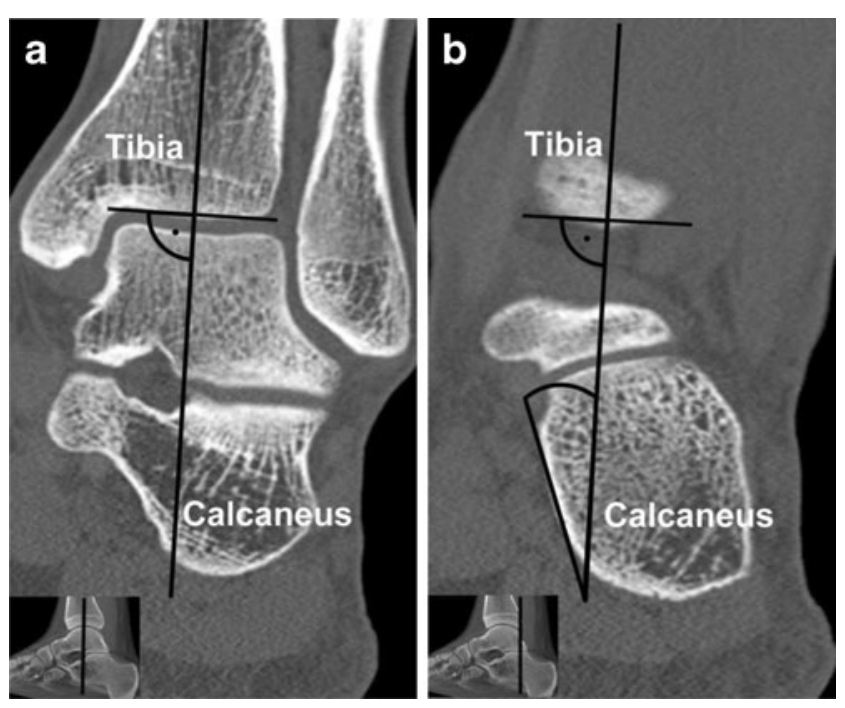

Fig. 2 A 25-year-old man with ankle joint pain. a and b Coronal reformations (1 $\mathrm{mm}$ slice thickness) of the ankle joint show the measurement technique for the hindfoot alignment angle. The axis of the distal tibia is defined by a perpendicular line to the midportion of the distal tibial joint surface (a). Measurement was obtained on the most posterior image including the tibia and calcaneus between the tibial axis and a line adapted to the medial osseous contour of the calcaneus (b)

\section{Statistical analysis}

Descriptive statistics were used to report quantitative data. Significant changes between NWBCT and WBCT were assessed using the Wilcoxon signed-rank test. Interreader agreement was quantified for all measurements using the intraclass correlation coefficient (ICC). According to Rosner [18], the interreader reliability by means of ICC is classified as follows: $>0.75$ is excellent, $0.4-0.75$ is fair to good, and $<0.4$
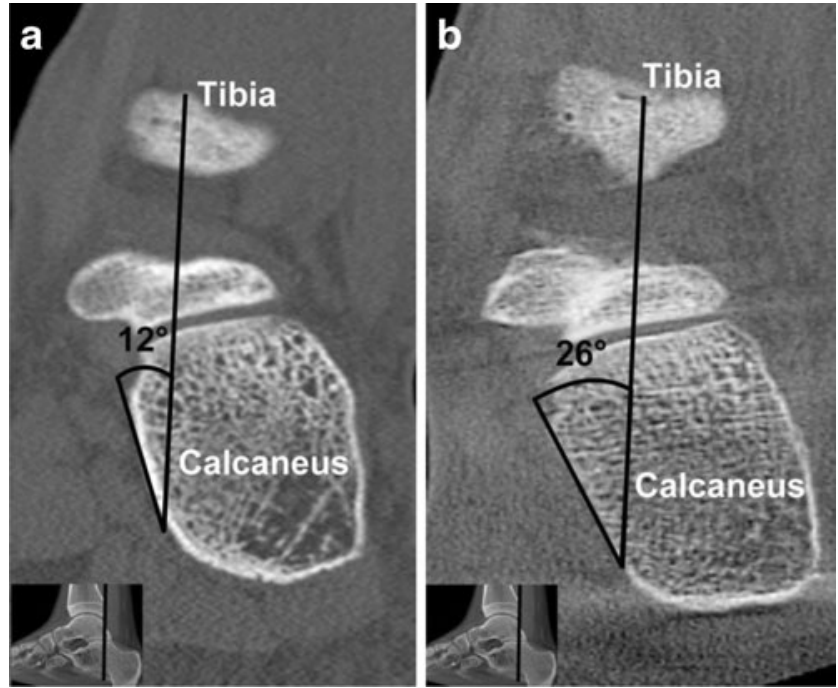

Fig. 3 A 25-year-old man with an osteochondral defect of the talus (not shown). Coronal reformation (1 mm slice thickness) in non-weight-bearing position (a) shows a hindfoot valgus of $12^{\circ}$; the hindfoot valgus increases to $26^{\circ}$ in the upright weight-bearing position (b) is poor. A $P$ value of $<0.05$ was considered statistically significant. For all analyses statistical software (SPSS for Windows, release 17.0; SPSS, Chicago, IL, USA) was used.

\section{Results}

Significant differences were found for all measurements except the hindfoot alignment angles and the tibiocalcaneal distances between NWBCT and WBCT (Table 1). The fibulocalcaneal distance decreased significantly in WBCT (reader $1 /$ reader 2 ; mean $0.3 \mathrm{~mm} /-1.1 \mathrm{~mm}$ ) compared to NWBCT $(3.6 \mathrm{~mm} / 1.4 \mathrm{~mm})$, whereas the negative value indicates the fibular tip below the calcaneal joint surface. The lateral talocalcaneal joint space width significantly decreased in WBCT $(2.2 \mathrm{~mm} / 2.4 \mathrm{~mm})$ compared to NWBCT $(2.9 \mathrm{~mm} /$ $3.4 \mathrm{~mm})$. The talocalcaneal overlap significantly decreased in WBCT $(1.4 \mathrm{~mm} / 1.4 \mathrm{~mm})$ compared to NWBCT $(4.1 \mathrm{~mm} /$ $4.5 \mathrm{~mm})$. The naviculocalcaneal distance increased significantly $(P=0.037)$ in WBCT $(15.3 \mathrm{~mm} / 15.7 \mathrm{~mm})$ compared to NWBCT $(13.5 \mathrm{~mm} / 14.0 \mathrm{~mm})$ for reader 1 (but only $P=$ 0.100 for reader 2 ).

Interreader agreement was excellent for the hindfoot alignment angle, lateral tibiocalcaneal and naviculocalcaneal distances, and talocalcaneal overlap in both positions (Table 2). The agreement was good for all other measurements.
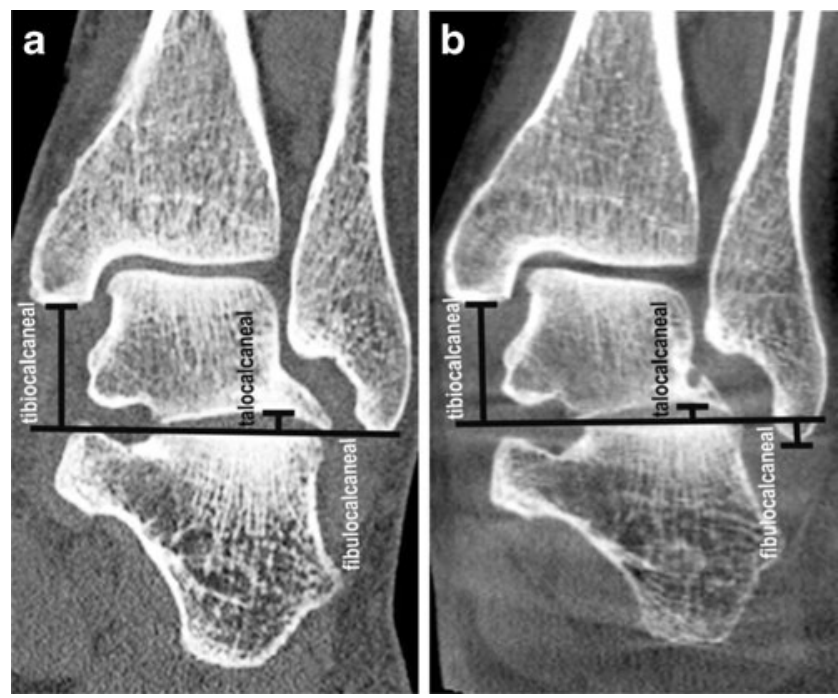

Fig. 4 A 50-year-old man with osteonecrosis of the navicular bone. Coronal image ( $1 \mathrm{~mm}$ slice thickness) in the non-weight-bearing position (a) demonstrates the fibular tip at the level of the reference line through the calcaneal surface of the posterior subtalar joint (fibulocalcaneal distance $=0 \mathrm{~mm}$ ). In the upright weight-bearing position $(\mathbf{b})$, the fibulocalcaneal distance decreases (fibulocalcaneal distance $=-7 \mathrm{~mm}$ ) with the fibular tip below the reference line. The lateral talocalcaneal distance decreases from $2.6 \mathrm{~mm}$ in NWBCT (a) to $1.8 \mathrm{~mm}$ in WBCT (b). The tibiocalcaneal distance increases from $15.9 \mathrm{~mm}$ in NWBCT (a) to $18.9 \mathrm{~mm}$ in WBCT (b) 

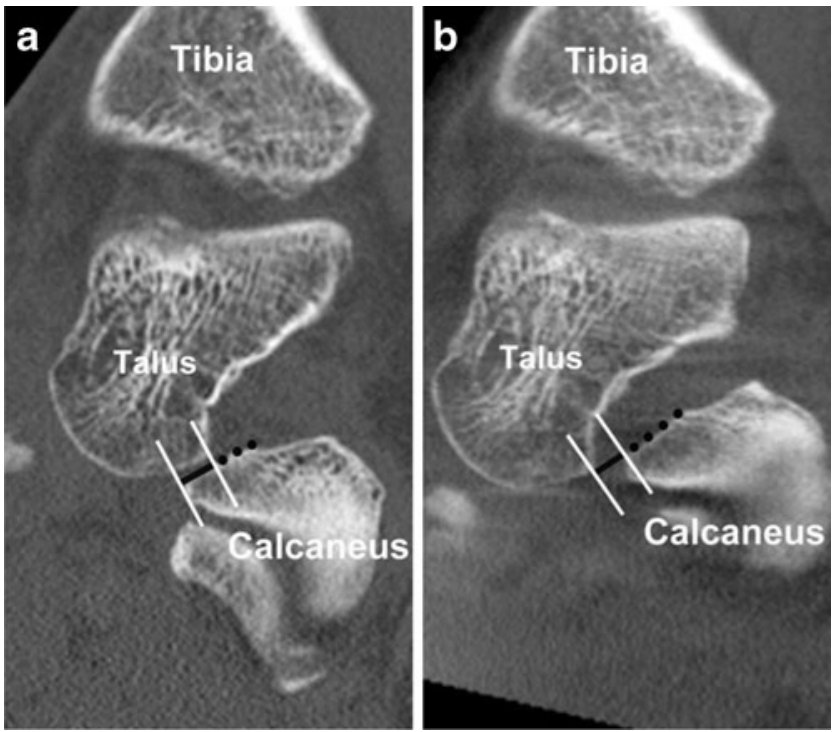

Fig. 5 A 50-year-old man with osteonecrosis of the navicular bone. Images demonstrate the measurement technique of the talocalcaneal overlap. In the non-weight-bearing (a) CT scan of the ankle with coronal reformation (1 mm slice thickness), the head of the talus overlaps the calcaneal facet of the anterior subtalar joint by $4.9 \mathrm{~mm}$. In the upright weight-bearing position (b), the talus does not overlap the calcaneal facet of the subtalar joint, resulting in a negative talocalcaneal overlap of $-4 \mathrm{~mm}$ (the dotted line represents the calcaneal facet)

\section{Discussion}

To date, hindfoot alignment has been evaluated using upright weight-bearing radiographs as well as CT and MR. Superimposition of the tarsal bones using X-ray limits a precise assessment of the hindfoot alignment [12]. Cross-sectional imaging is superior to radiographs in the evaluation of the hindfoot alignment and has been widely used for the assessment of feet deformities and ankle impingement; however, CT and MR have been restricted to a supine position $[2-4,6,12$, $16,17]$. The novel cone-beam extremity CT allows upright weight-bearing scans of the lower extremity because of a new scanner design $[13,14]$. This technique provides multiplanar reformations of CT images of the anatomy of the foot under physiological, weight-bearing conditions. Comparison of hindfoot alignment in NWBCT and WBCT has been presented. Significant differences in the weight-bearing position were found for the fibulocalcaneal distance, lateral talocalcaneal joint space width, naviculocalcaneal distance and talocalcaneal overlap. The ICC values in NWBCT and upright WBCT reached values close to 1 , demonstrating the excellent reproducibility of the measurement methods.

The significant decrease in the fibulocalcaneal distance in the weight-bearing position might be due to the tendency of pronounced hindfoot valgus compared to the non-weightbearing position, although the hindfoot alignment angle did not show a significant difference $(P=0.249$ and 0.581 for reader 1 and 2, respectively). In the weight-bearing position the tibiocalcaneal joint space width decreased significantly, leading to a decrease in the fibulocalcaneal distance. The talocalcaneal distance merely showed a decreasing tendency in the weight-bearing CT for reader 1.

Due to substantial limitations in the evaluation of the hindfoot using X-rays [12], we assessed the hindfoot alignment angle, the fibulocalcaneal and tibiocalcaneal distances, the lateral talocalcaneal joint space width and the talocalcaneal overlap in the coronal plane as well as the naviculocalcaneal distance in the sagittal plane using CT. Using multiplanar reformations and volume reconstructions, the joint surfaces of the foot can confidently be detected and reference lines can be defined accurately for measurements $[3,4,7,8,12-14]$. Furthermore, radiographic evaluation of hindfoot alignment is prone to misleading measurement results because of malpositioning of the foot at the time of image acquisition and may be limited in assessing the source of foot instability $[2,12]$. Such problems can be excluded by using CT with secondary multiplanar reconstructions.

To date, several authors have investigated foot alignment with simulated weight-bearing CT in the supine position using special apparatuses [6-9]. The weight-load differed between 10 and $50 \%$ of the body weight $[6,7]$. Imaging under partial weight-load seems to underestimate subluxation of the foot joints and minor changes of the joint alignment may remain undiscovered [6]. So far, only Kido et al. [8, 9] have simulated weight-bearing CT scans of the foot in the supine position with full-body weight-load applied to a knee plate. However, the supine position may eliminate the pull of the gastrocnemius muscle and again diminish subluxation in the foot joint

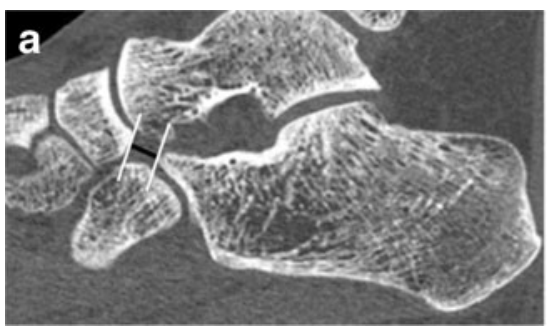

Fig. 6 A 36-year-old woman with an osteochondral defect of the talus (not shown). Sagittal images (2 mm slice thickness) of a $\mathrm{CT}$ scan of the ankle joint show the measurement technique of

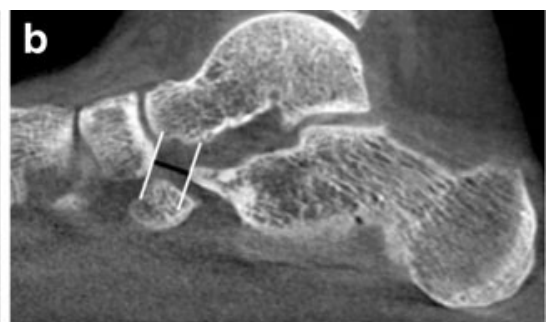

the naviculocalcaneal distance. The distance increases in the weight-bearing position (b) compared to the non-weight bearing position (a) 
Table 1 Quantitative analysis of hindfoot alignment in non-weight-bearing CT (NWBCT) and upright weight-bearing computed tomography (WBCT)

\begin{tabular}{|c|c|c|c|c|c|c|}
\hline & \multicolumn{3}{|l|}{ Reader 1} & \multicolumn{3}{|l|}{ Reader 2} \\
\hline & NWBCT & WBCT & $P$ value & NWBCT & WBCT & $P$ value \\
\hline Hindfoot alignment angle $\left(^{\circ}\right)$ & $19.0 \pm 9.0$ & $21.0 \pm 7.9$ & 0.249 & $19.7 \pm 9.0$ & $20.8 \pm 10.0$ & 0.581 \\
\hline Fibulocalcaneal distance (mm) & $3.6 \pm 5.2$ & $0.3 \pm 6.0$ & $0.006^{*}$ & $1.4 \pm 6.3$ & $-1.1 \pm 6.3$ & $0.002 *$ \\
\hline Tibiocalcaneal distance (mm) & $21.7 \pm 6.2$ & $20.6 \pm 4.2$ & 0.313 & $21.6 \pm 6.7$ & $21.8 \pm 6.6$ & 0.627 \\
\hline Lateral talocalcaneal joint space width (mm) & $2.9 \pm 1.7$ & $2.2 \pm 1.1$ & $0.005^{*}$ & $3.4 \pm 1.9$ & $2.4 \pm 1.3$ & $0.001 *$ \\
\hline Talocalcaneal overlap(mm) & $4.1 \pm 3.9$ & $1.4 \pm 3.9$ & $0.001 *$ & $4.5 \pm 4.3$ & $1.4 \pm 3.7$ & $<0.001^{*}$ \\
\hline Naviculocalcaneal distance (mm) & $13.5 \pm 4.0$ & $15.3 \pm 4.7$ & $0.037^{*}$ & $14.0 \pm 4.4$ & $15.7 \pm 6.2$ & 0.100 \\
\hline
\end{tabular}

Data are mean values with standard deviations in parentheses

Positive values of hindfoot alignment angle represent valgus. Negative values represent the following: hindfoot varus, fibular tip below the superior calcaneal joint surface and absence of talocalcaneal overlap

$* P<0.05$ was considered statistically significant

[6]. The upright, weight-bearing position exclusively enables imaging under physiological conditions of the foot joints and allows a precise evaluation of the joint alignment.

So far, comparison of foot alignment has mainly been accomplished using special software packages with the need for segmentation of the tarsal bones and analyses of tarsal bone rotation in $3 \mathrm{D}$ images $[6,8,9]$. The aim of our study was to demonstrate the change in hindfoot alignment in upright weight-bearing CT compared to supine non-weight-bearing $\mathrm{CT}$ in the coronal and sagittal planes for daily purposes in the clinical setting without requiring special software. Therefore, we carried out measurements of the hindfoot, such as the fibulocalcaneal and tibiocalcaneal distances, the lateral talocalcaneal joint space width, the naviculocalcaneal distance and the talocalcaneal overlap, which have not been described in the current literature before. However, our findings of a significant decrease in the fibulocalcaneal distance and the talocalcaneal overlap in WBCT compared to NWBCT are comparable with the results of Ferri et al. [7]. They found a subtalar joint subluxation and an increased tarsal bone shift in

Table 2 Interreader agreement of hindfoot alignment in non-weightbearing $\mathrm{CT}$ (NWBCT) and upright weight-bearing computed tomography (WBCT)

\begin{tabular}{lll}
\hline & NWBCT & WBCT \\
\hline Hindfoot alignment angle & 0.87 & 0.83 \\
Fibulocalcaneal distance & 0.48 & 0.61 \\
Tibiocalcaneal distance & 0.67 & 0.72 \\
Lateral talocalcaneal joint space width & 0.83 & 0.82 \\
Talocalcaneal overlap & 0.94 & 0.81 \\
Naviculocalcaneal distance & 0.79 & 0.85 \\
\hline
\end{tabular}

Intraclass correlation coefficient (ICC) according to Rosner (15): $>0.75$ excellent, $0.4-0.75$ fair to good, $<0.4$ poor pes planus feet, which was more evident in simulated weightbearing CT compared to non-weight-bearing CT [7]. Only the hindfoot alignment angle has been established in crosssectional imaging before $[16,17,19]$. The normal hindfoot valgus angle was defined as less than $6^{\circ}$ in early years [20]. Donovan et al. [16] questioned this reference value. They measured higher values of hindfoot valgus of $7-26^{\circ}$ in the majority of patients with impingement of the hindfoot. In our study we measured a mean hindfoot valgus of $20^{\circ}$; this is in accordance with the findings of Donovan et al. [16]. Any hindfoot varus is reported to be abnormal [17], but we did not observe this finding in our study population.

Proper evaluation of hindfoot alignment is crucial to assess the amount of congenital or acquired foot deformity, such as pes planovalgus and ankle impingement, for example $[2-4,6,16]$. In unloaded, non-weight-bearing scans, the impingement configuration may be underestimated or even undiagnosed; therefore, weight-bearing assessment of the foot seems necessary.

Upright WBCT facilitates a diagnostic procedure for the ankle and foot and precisely demonstrates the hindfoot alignment in full-body weight-bearing conditions. MR imaging is a widely used imaging method in the evaluation of foot pain or deformity, especially in assessing the soft tissue $[16,17,19]$. It lacks the possibility of upright weight-bearing imaging of the foot $[16,17]$, although this is possible using upright MR systems. The bony structures and joint alignment can be precisely demonstrated using $\mathrm{CT}$.

The present study has several limitations. The clinical indications varied, resulting in a heterogeneous patient population. The number of patients is rather low because of the exclusion criteria in a fully weight-bearing examination of the foot (postoperative, early posttraumatic, wheel-chair bound). However, as this is a novel CT system allowing fully weightbearing imaging for the first time, further investigations are required with healthy volunteers to demonstrate the normal range of differences in non-weight-bearing and weight- 
bearing foot alignments and to evaluate a larger number of patients in order to define accurate measurement thresholds. In conclusion, alignment of the hindfoot significantly changes in upright weight-bearing position. Differences can be well visualised and measured using weight-bearing cone-beam computed tomography.

\section{References}

1. Nosewicz TL, Knupp M, Bolliger L, Hintermann B (2012) The reliability and validity of radiographic measurements for determining the three-dimensional position of the talus in varus and valgus osteoarthritic ankles. Skeletal Radiol 41:1567-1573

2. Buck FM, Hoffmann A, Mamisch-Saupe N, Espinosa N, Resnick D, Hodler J (2011) Hindfoot alignment measurements: rotation-stability of measurement techniques on hindfoot alignment view and long axial view radiographs. AJR Am J Roentgenol 197:578-582

3. Van Bergeyk AB, Younger A, Carson B (2002) CT analysis of hindfoot alignment in chronic lateral ankle instability. Foot Ankle Int 23:37-42

4. Greisberg J, Hansen ST, Sangeorzan B (2003) Deformity and degeneration in the hindfoot and midfoot joints of the adult acquired flatfoot. Foot Ankle Int 24:530-534

5. Saltzmann CL, el-Khoury GY (1995) The hindfoot alignment view. Foot Ankle Int 16:572-576

6. Ananthakrisnan D, Ching R, Tencer A, Hansen ST, Sangeorzan BJ (1999) Subluxation of the talocalcaneal joint in adults who have symptomatic flatfoot. J Bone Joint Surg Am 81A:1147-1154

7. Ferri M, Scharfenberger AV, Goplen G, Daniels TR, Pearce D (2008) Weightbearing CT scan of severe flexible pes planus deformity. Foot Ankle Int 29:199-204

8. Kido M, Ikoma K, Imai K, Maki M, Takatori R, Tokunaga D et al (2011) Load response of the tarsal bones in patients with flatfoot deformity: in vivo 3D study. Foot Ankle Int 32:1017-1022
9. Kido M, Ikoma K, Imai K, Inoue N, Kubo T (2013) Load response of the medial longitudinal arch in patients with flatfoot deformity: in vivo 3D study. Clin Biomech 28:568-573

10. Ledoux WR, Rohr ES, Ching RP, Sangeorzan BJ (2006) Effect of foot shape on the three-dimensional position of foot bones. J Orthop Res 24:2176-2186

11. Malicky ES, Crary JL, Houghton MJ, Agel J, Hansen ST, Sangeorzan BJ (2002) Talocalcaneal and subfibular impingement in symptomatic flatfoot in adults. J Bone Joint Surg Am 84A:2005-2009

12. Ellis SJ, Deyer T, Williams BR, Yu JC, Lehto S, Maderazo A, Pavlov $\mathrm{H}$ et al (2010) Assessment of lateral hindfoot pain in acquired flatfoot deformity using weightbearing multiplanar imaging. Foot Ankle Int 31:361-371

13. Tuominen EKL, Kankare J, Koskinen SK, Mattila KT (2013) Weight-bearing CT imaging of the lower extremity. AJR Am J Roentgenol 200:146-148

14. Koskinen SK, Haapamäki VV, Salo J, Lindfors NC, Kortesniemi M, Seppälä L, Mattila KT (2013) CT arthrogarphy of the wrist using a novel, mobile, dedicated extremity cone-beam CT (CBCT). Skeletal Radiol 42:649-657

15. Ippolito E, Fraracci L, Farsetti P, De Maio F (2004) Validity of the anteroposterior talocalcaneal angle to assess congenital clubfoot correction. AJR Am J Roentgenol 182:1279-1282

16. Donovan A, Rosenberg ZS (2009) Extraarticular lateral hindfoot impingement with posterior tibial tendon tear: MRI correlation. AJR Am J Roentgenol 193:672-678

17. Buck FM, Hoffmann A, Mamisch-Saupe N, Farshad M, Resnick D, Espinosa N, Hodler J (2013) Diagnostic performance of MRI measurements to assess hindfoot malalignment. An assessment of four measurement techniques. Eur Radiol 23:2594-2601

18. Rosner BA (2011) The intraclass correlation coefficient. In: Fundamentals of Biostatistics, 7th edn. Brooks/Cole, Boston, USA, p 569

19. Donovan A, Rosenberg ZS (2010) MRI of ankle and lateral hindfoot impingment syndromes. AJR Am J Roentgenol 195:595-604

20. Seltzer SE, Weissmann BN, Braunstein EM, Adams DF, Thomas WH (1984) Computed tomography of the hindfoot. J Comput Assist Tomogr 8:488-497 